\title{
The relationship between emotional appetite and bipolar features in obese and non-obese individuals
}

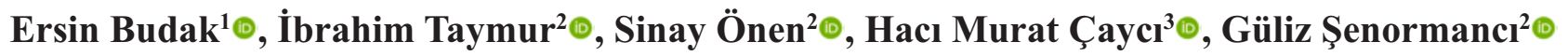 \\ ${ }^{1}$ Department of Psychology, University of Health Sciences, Bursa Yüksek Ihtisas Training and Research Hospital, Bursa, Turkey \\ ${ }^{2}$ Department of Psychiatry, University of Health Sciences, Bursa Yüksek Ihtisas Training and Research Hospital, Bursa,, Turkey \\ ${ }^{3}$ Department Of General Surgery, University of Health Sciences, Bursa Yüksek Ihtisas Training and Research Hospital, Bursa, Turkey
}

DOI: 10.18621 /eurj.433962

\begin{abstract}
Objectives: It is known that many different positive and negative emotions can affect appetite and also, individuals who have bipolar features often have emotional fluctuations. In this study, it was aimed to investigate the relationship between emotional appetite and bipolar features in obese individuals.

Methods: One hundred and ninety obese individuals who applied for bariatric surgery and 136 non-obese individuals were evaluated with Emotional Appetite Questionnaire (EMAQ), Beck- Depression Inventory (BDI), Beck-Anxiety Inventory (BAI) and The Temperament Evaluation of Memphis, Pisa, Paris and SanDiego Auto-questionnaire (TEMPS-A) in the study.

Results: In obese individuals who applied for bariatric surgery, the frequency of bipolar disorder was found to be $2 \%$ and binge eating disorder (BED) frequency was $51.2 \%$. It was found that scores of appetite in negative emotions were higher in obese individuals with BED compared to obese individuals and it was higher in obese individuals compared to normal weight individuals. Cyclothymic features explained $27.2 \%, 25.8 \%$ and $15.7 \%$ of scores of appetite in negative situations of obese individuals with BED, obese individuals without BED and normal weight individuals, respectively.

Conclusions: As a result of this study, it can be concluded that scores of appetite in negative situations may be affected by cyclothymic features in obese individuals with BED, in obese and normal weight individuals. Depression and anxiety symptoms are effective factors in explaining scores of appetite in negative situations of obese individuals with BED, obese and normal weight individuals.
\end{abstract}

Keywords: emotional appetite, bipolar features, obesity, cyclothymia

Received: June 14, 2018; Accepted: August 7, 2018; Published Online: January 21, 2019

E motional appetite is defined as increase in the consumption of food in order to cope with unpleasant feelings and situations [1]. There are data indicating that emotional appetite may be related to individuals' body mass index (BMI) and emotional appetite may cause obesity $[1,2]$. Furthermore, emotional appetite has been shown to be an effective factor in the relationship between eating disorders (binge eating disorder [BED] and bulimia nervosa) and obesity [3-5]. The presence of a relationship between obesity, eating disorders and emotional appetite has caused more research into emotional appetite. Consequently, Nolan et al. [2] defined appetite changes caused by psychological symptoms as a construct related to neg-

Address for correspondence: Ersin Budak, Psychologist, University of Health Sciences, Bursa Yüksek Ihtisas Training and Research Hospital, Department of Psychiatry, Bursa, Turkey 
ative and positive emotional states. In this study, it was found that compared to their usual eating habits, overweight individuals reported to consume more food in negative situations than underweight individuals, and underweight individuals reported to consume more food in positive emotions than overweight individuals.

BED is defined as an eating disorder characterized by recurrent periods of binge eating with the sense of loss of control over eating behavior [6]. It has been argued that problematic eating behaviors and emotional appetite are related in individuals diagnosed with BED [4]. In a study of obese women, emotional appetite was suggested to be one of the determinants of BED [7]. It has been indicated that emotional regulation problemshave significant effects on emotional overeating and eating pathologies in individuals with BED [8]. In studies conducted on individuals with a desire to lose weight, it was reported that individuals with BED could be experiencing serious problems in appetite control $[9,10]$. Frequency of emotional appetite symptoms have also been found to be high in individuals with a desire to lose weight [11]. In a study comparing participants who had weight loss with bariatric surgery with participants who had diet-induced weight loss, it was shown that neurotic features were effective in weight loss in both groups and emotional appetite mediated this effect, and in both groups overeating behavior was decreased [12]. In another study conducted on post-bariatric surgery patients, obese patients were evaluated to have positive change in emotional appetite as their weight decreased [13].

Bipolar and related disorders are defined as mental disorders with manic, hypomanic and depressive symptoms. In DSM 5, bipolar diagnoses (bipolar I, bipolar II, cyclothymia, other specified, unspecified bipolar and related disorder) are classified according to the severity, frequency, duration and content of manic, hypomanic and depressive symptoms [14]. Studies have revealed that eating disorders are prevalent in individuals with bipolar disorder, at the same time bipolar disorder comorbidity is high among individuals with eating disorders [15]. In a study on young patients with bipolar disorder, patients with bipolar disorder were evaluated as more likely to exhibit overeating and emotional eating behaviors compared with individuals without bipolar disorder [16]. In a study investigating psychiatric diagnoses of individuals applying for bariatric surgery, current bipolar dis- order rate was $13.7 \%$ and lifetime prevalence was $35.6 \%$ [17]. In another study evaluating 120 consecutive participants seeking bariatric surgery, bipolar disorder frequency was found to be $91.7 \%$ [18]. Despite these studies, in a study conducted on 935 obese patients, bipolar disorder rate was reported as $6 \%$, still, this bipolar disorder ratio was higher than those of normal population [19].

Research on emotional appetite in obese individuals focus on eating disorders, depression, anxiety, anger and neurotic features $[2,20,21]$. Nevertheless, there are no studies in the literature investigating the relationship between bipolar features and emotional appetite in obese individuals. It is known that there are many different emotions effective in emotional appetite [2], including positive (confident, happy, relaxed, playful) and negative emotions (sad, bored, angry, anxious, etc) and individuals with bipolar features often have emotional fluctuations and rapid shifts in mood [14]. It is stated that especially in individuals with cyclothymic features, major emotional changes are seen during the day [22] and at the same time, appetite can be affected when individuals are in negative (when under pressure, after a heated argument, etc) and positive situations (when falling in love, after receiving good news, etc) [2]. Therefore, it was aimed to investigate the relationship between emotional appetite and bipolar features in obese individuals.

\section{METHODS}

\section{Participants}

Two hundred and eleven voluntary obese individuals (BMI > 34.9) who have applied for bariatric surgery between November 2013 and February 2016 were invited to participate in the study. Twelve individuals refused to participate in the study. Exclusion criteria of the study was having any mental or organic disease, psychotic disorder, bipolar disorder or bulimia nervosa. Before including the study, patients were evaluated if they had any psychiatric disorders that prevented them from participating in the study. Consequently, of 199 morbidly obese participants, 4 (2\%) had diagnosis of bipolar disorder (3 of them bipolar I, 1 of bipolar II), $1(0.5 \%)$ had psychotic disorder, $102(51.2 \%)$ had BED and 4 (2\%) had bulimia nervosa. Participants with a diagnosis of 
bipolar disorder, bulimia nervosa, or of any psychiatric disorder that prevents from participating the study were excluded. So, in this study, nonpathological bipolar features were investigated in obese and normal weight individuals. Obese patients were divided into two groups according to having BED diagnosis. By this means, we attempted to control the confounding effect of this diagnosis on emotional appetite. The reason for this is that studies indicate that BED diagnosis and BED features can affect emotional appetite $[4,7,8]$.

To compare data of obese individuals, we wanted to design a control group of non-obese individuals (18 $<$ BMI < 30) with similar sociodemographic (age, education level, sex) characteristics with obese individuals. For this purpose, 149 individuals who applied to the family medicine department of our hospital were invited to participate in the study. The exclusion criteria in non-obese group was having a diagnosis of any organic or mental disorder, psychotic disorder, bipolar disorder or bulimia nervosa. In addition, a history of obesity (BMI $>30$ ) or of having bariatric surgery were defined as other exclusion criteria. For this reason, 11 participants with a history of obesity and 2 participants with BED were excluded from the study. In both obese and control group, psychiatric diagnosis and exclusion criteria were evaluated by clinical interview. Consequently, psychometric data of 102 obese with BED, 88 obese without BED and 136 non-obese participants who were eligible according to exclusion criteria were examined.

\section{Measures}

Emotional Appetite Questionnaire (EMAQ) was used to assess emotional appetite of participants. Beck-Depression Inventory (BDI), Beck-Anxiety Inventory (BAI) and The Temperament Evaluation of Memphis, Pisa, Paris and San-Diego Auto questionnaire (TEMPS-A) were used to evaluate bipolar features. Besides these, all participants were given sociodemographic data form designed by the researchers including questions about age, sex, height, weight, past psychiatric admissions.

\section{Emotional Appetite}

EMAQ was developed by Nolan et al. [2] in 2010. The presence of emotional eating is evaluated in negative/positive emotions (14 items) and in negative/positive situations ( 8 items). The validity study of Turkish version of the Cronbach's alpha coefficient was 0.73 and the inter-item correlation coefficients of the scale were between 0.14-0.72 [23]. Every item in the scale is scored between -4 and +4 and there are four subscales in the scale as positive emotions (EMAQ-PE), negative emotions (EMAQ$\mathrm{NE}$ ), positive situations (EMAQ-PS) and negative situations (EMAQ-NS). High scores from the subscales of the scale indicate high level of emotional and situational appetite features.

\section{Depression}

BDI, which was developed by Beck et al. [24] to determine the severity of the depressive symptoms of an individual, was adapted to Turkish by Hisli [25]. This scale, consisting of 21 items, is evaluated based on a spectrum of 0 to 63 points, and its Cronbach's alpha coefficient is 0.80 . It is used to assess the depression symptoms experienced during past week. The higher the score obtained on the scale indicates the more severe depressive symptoms the individual is experiencing.

\section{Anxiety}

BAI which was developed by Beck et al. [26] to determine the severity of the anxiety symptoms of an individual, was adapted to Turkish by Ulusoy et al. [27]. The correlation coefficients of the scale were between 0.45-0.72 and the Cronbach's alpha value was 0.93 . This scale, consisting of 21 items, is evaluated based on a total score that ranges from 0 to 63. It is used to evaluate anxiety symptoms seen during past week. The higher the score obtained from the scale, the more severe anxiety the individual is experiencing.

\section{Bipolar Features}

Memphis, Pisa, Paris and San-Diego Autoquestionnaire (TEMPS-A) is a likert type of scale developed by Akiskal et al. [22]. It is a measure used to evaluate major and stable traits that are effective in emotional variations of individuals with and without bipolar disorder. Its Turkish version and validity and reliability study were done by Vahip et al. [28]. Five sub-temperament features are measured, which are cyclothymic, depressive, hyperthymic, irritable and 
anxious. The test-re-test reliability of the Turkish version is between 0.73 and 0.93 , and the Cronbach's alpha coefficient is between 0.75 and 0.84 . Cyclothymic temperament involves emotional fluctuations (I get sudden shifts in mood and energy, my mood often changes for no reason) and polarity of emotions (My moods and energy are either high or low, rarely in between). Depressive temperament involves hopelessness, sadness (I am a sad, unhappy person) and lack of energy (I do not seem to have as much energy as other people). Hyperthymic temperament involves cheerful, optimistic, enthusiastic features (I am usually in an upbeat or cheerful mood). Irritable temperament is associated with pessimistic, grumpy, dissatisfied and irritable states (I am a grouchy person, I am by nature a dissatisfied person). Anxious temperament involves fear and anxiety (I am often fearful of someone in my family coming down with a serious disease), somaticcomplaints (When I am nervous, I often feel nauseous).

\section{Procedure}

Voluntary participants were recruited in the study. Prior to participation in the study, an informed consent form was signed by all participants. After evaluation of the psychiatric diagnoses, participants were individually assessed by sociodemographic data form and psychometric scales. DSM-5 was used in the evaluation of psychiatric diagnoses (mental disorders, bipolar and related disorders, BED, bulimia nervosa, psychosis). Approval from the ethics committee was obtained. In addition, the study was conducted according to the Declaration of Helsinki.

\section{Statistical Analysis}

Pearson correlation analysis was used to analyze the relationship between EMAQ, BDI, BAI and TEMPS-A in obese and non-obese individuals. Chisquare test was used to compare the ratios related to sociodemographic data of the individuals in the control group and obese patients. One Way ANOVA was used to compare the average scores of EMAQ, BDI, BAI and TEMPS-A between the obese with BED, obese without BED and control group. In addition the Tukey test was used to determine which groups differed from each other in the mean scores. Hierarchical regression analysis was used to measure

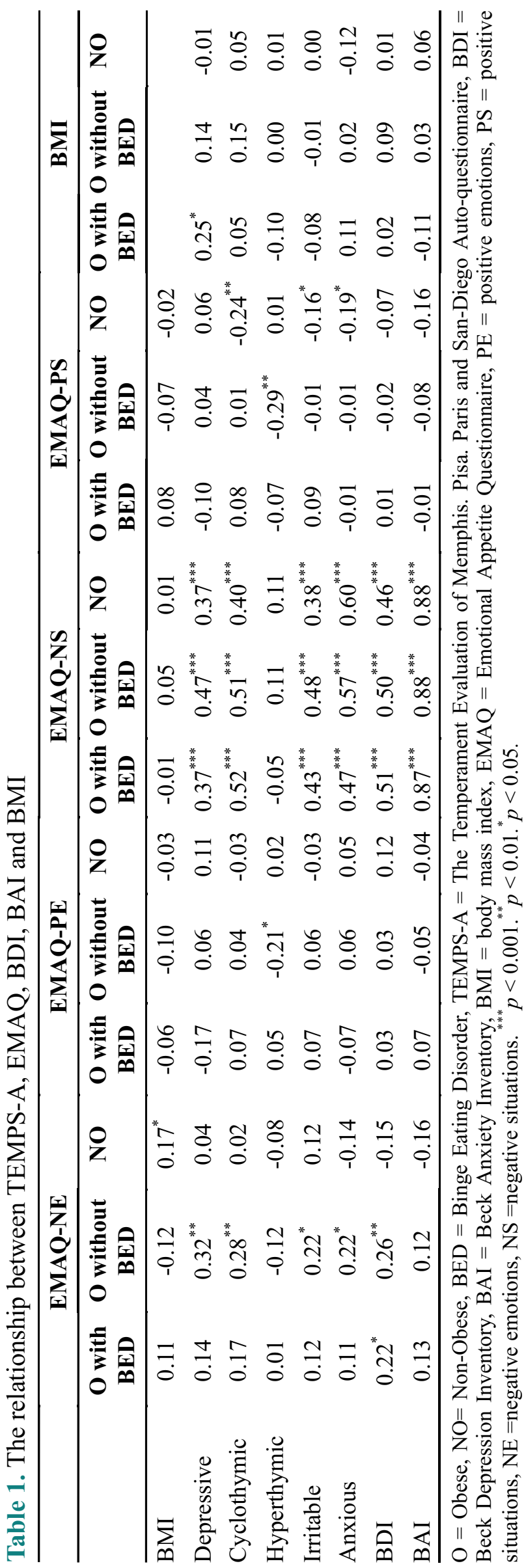


whether cyclothymic, depressive, hyperthymic, irritable and anxious temperaments scores were predicted by the abnormal EMAQ scores. Significance level for all the analyses was determined as $p<0.05$. IBM SPSS Statistics 22.0 software was used in analyzing the data. Normal distribution assumptions were met for the Pearson correlation analysis, One Way ANOVA and Hierarchical Regression Analysis.

\section{RESULTS}

Obese patients between the ages of 18-68 were included in our study. The age range of the healthy control group was 20-62. Mean age of obese individuals with BED (34.44 \pm 8.48 years), obese (36.77 \pm 10.27 years) and normal weight $(34.19 \pm 8.6$ years) individuals did not differ statistically $(\mathrm{F}=2.41$, $p=0.091) .21(20.6 \%)$ of obese with BED, $17(19.3 \%)$ of obese, $34(25 \%)$ of normal weight individuals were male and sex ratios were similar for the three groups $\left(\mathrm{X}^{2}=1.96, p=0.550\right) .40(20.6 \%)$ of obese with BED, $20(19.3 \%)$ of obese, $23(25 \%)$ of normal weight individuals had a history of psychiatric treatment and psychiatric treatment history ratios were statistically differentfor the three groups (X2 $=15.75, p<0.001)$. A positive correlation was found between EMAQ$\mathrm{NE}$ scores and BMI in non-obese participants $(\mathrm{r}=$ $0.17, p<0.05)$. It was found that there was a positive relationship between EMAQ-NE scores and depressive $(\mathrm{r}=0.32, p<0.01)$, cyclothymic $(\mathrm{r}=0.28$, $p<0.001)$, irritable $(\mathrm{r}=0.22, p<0.05)$, anxious temperament scores $(\mathrm{r}=0.22, p<0.05)$ in obese participants. A positive correlation was found between EMAQ-NE scores and BDI scores in obese participants $(\mathrm{r}=0.26, p<0.01)$. There was a positive relationship only between EMAQ-NE and BDI in obese participants with BED $(r=0.22, p<0.05)$. In the three groups, there was a negative relationship between EMAQ-PE scores and hyperthymic temperament in only obese individuals $(\mathrm{r}=-0.21, p<$ $0.05)$. There was a positive relationship ranging from 0.37-0.88 between EMAQ-NS scores and depressive, cyclothymic, irritable and anxious temperament, BDI, BAI scores in the three groups. There was a negative relationship between EMAQ-PS scores and cyclothymic temperament $(\mathrm{r}=-0.24, p<0.01)$, irritable temperament $(\mathrm{r}=-0.16, p<0.05)$, anxious temperament $(\mathrm{r}=-0.19, p<0.05)$ in non-obese group. There was a negative relationship between EMAQ-PS

Table 2. Comparison of results of the TEMPS-A, EMAQ, BDI and BAI between the obese and non-obese group

\begin{tabular}{lccccc}
\hline & O with BED & O without BED & NO & F & p value \\
\hline Depressive $^{\mathrm{a}}$ & $7.64 \pm 3.29$ & $6.61 \pm 3.30$ & $5.56 \pm 3.30$ & 11.66 & $<0.001$ \\
Cyclothymic $^{\mathrm{b}}$ & $9.90 \pm 4.33$ & $8.30 \pm 4.84$ & $7.08 \pm 4.42$ & 11.31 & $<0.001$ \\
Hyperthymic $^{\mathrm{b}}$ & $11.72 \pm 4.00$ & $11.55 \pm 3.51$ & $9.95 \pm 3.87$ & 7.80 & $<0.001$ \\
Irritable $^{\mathrm{c}}$ & $3.97 \pm 3.49$ & $3.63 \pm 3.80$ & $3.13 \pm 3.38$ & 1.69 & 0.186 \\
Anxious $^{\mathrm{a}}$ & $7.29 \pm 4.56$ & $6.22 \pm 4.94$ & $5.21 \pm 4.18$ & 6.20 & 0.002 \\
BDI $^{\mathrm{b}}$ & $15.14 \pm 8.08$ & $12.87 \pm 8.20$ & $8.21 \pm 7.61$ & 23.75 & $<0.001$ \\
BAI $^{\mathrm{b}}$ & $14.66 \pm 10.24$ & $13.37 \pm 7.60$ & $6.49 \pm 7.23$ & 25.79 & $<0.001$ \\
EMAQ-NE $^{\mathrm{d}}$ & $2.02 \pm 12.42$ & $-7.35 \pm 13.98$ & $-12.86 \pm 12.53$ & 38.93 & $<0.001$ \\
EMAQ-PE $^{\mathrm{c}}$ & $1.12 \pm 6.43$ & $-0.38 \pm 6.23$ & $1.47 \pm 5.01$ & 2.88 & 0.057 \\
EMAQ-NS $^{\mathrm{b}}$ & $3.15 \pm 2.77$ & $2.69 \pm 2.89$ & $1.20 \pm 1.94$ & 19.95 & $<0.001$ \\
EMAQ-PS $^{\mathrm{c}}$ & $-1.01 \pm 5.19$ & $-1.60 \pm 4.87$ & $-0.41 \pm 4.18$ & 1.73 & 0.178 \\
BMI $^{\mathrm{e}}$ & $46.29 \pm 5.47$ & $46.90 \pm 5.21$ & $22.56 \pm 2.60$ & 1126.43 & $<0.001$ \\
\hline
\end{tabular}

$\mathrm{O}=$ Obese, $\mathrm{NO}=$ Non-Obese, BED =Binge Eating Disorder, TEMPS-A $=$ The Temperament Evaluation of Memphis. Pisa. Paris and San-Diego Auto-questionnaire, BDI = Beck Depression Inventory, BAI = Beck Anxiety Inventory, EMAQ = Emotional Appetite Questionnaire, $\mathrm{PE}=$ positive emotions, $\mathrm{PS}=$ positive situations, $\mathrm{NE}=$ negative emotions, $\mathrm{NS}=$ negative situations. ${ }^{\mathrm{a}} \mathrm{NO}<\mathrm{O}$ with $\mathrm{BED},{ }^{\mathrm{b}} \mathrm{NO}, \mathrm{O}$ without $\mathrm{BED}<\mathrm{O}$ with $\mathrm{BED},{ }^{\mathrm{c}} \mathrm{NO}, \mathrm{O}$ without $\mathrm{BED}$, $\mathrm{O}$ with $\mathrm{BED},{ }^{\mathrm{d}} \mathrm{NO}<\mathrm{O}$ without $\mathrm{BED}<\mathrm{O}$ with $\mathrm{BED},{ }^{\mathrm{e}} \mathrm{NO}<\mathrm{O}$ without $\mathrm{BED}$, $\mathrm{O}$ with $\mathrm{BED}$. 


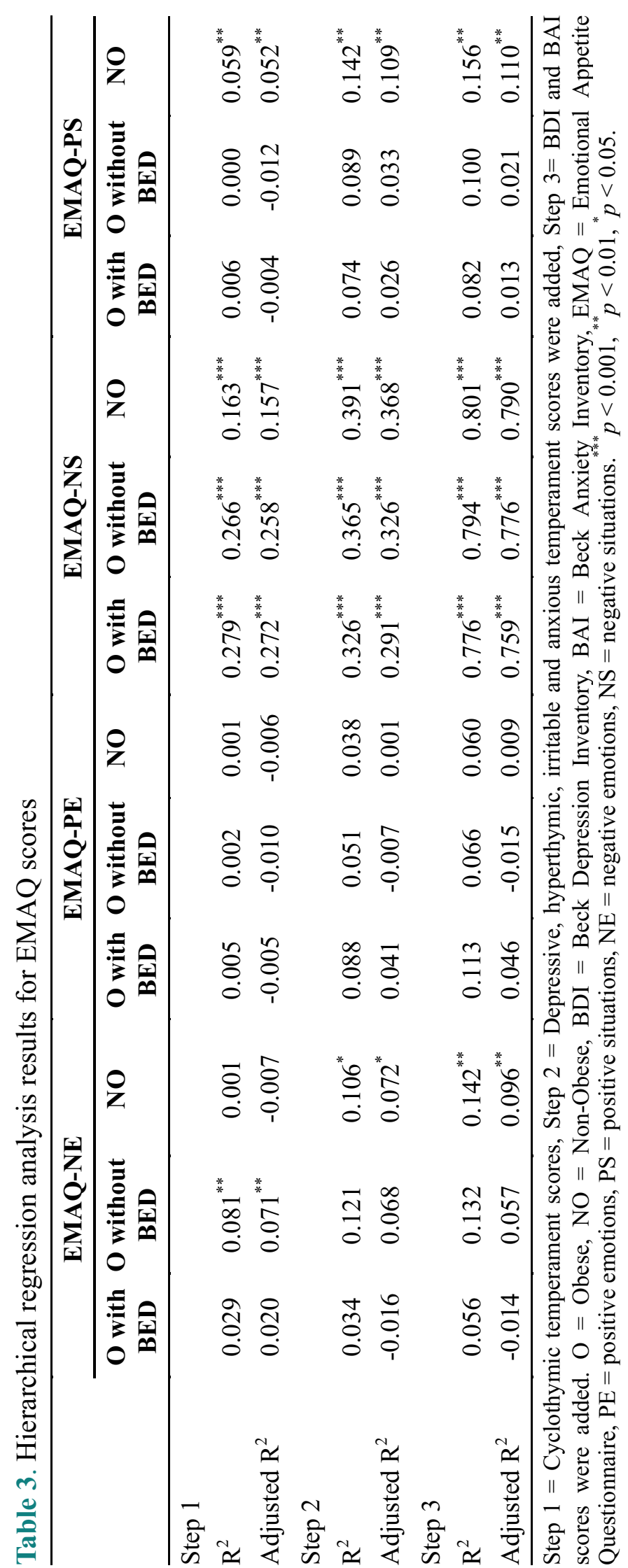

scores and hyperthymic temperament scores in obese group $(r=-0.29, p<0.01)$. Among the three groups, there was a negative relationship between BMI and depressive temperament scores in only obese individuals with $\mathrm{BED}(\mathrm{r}=0.25, p<0.05)$ (Table 1$)$.

Depressive temperament ( $p<0.001)$, cyclothymic temperament $(p<0.001)$, hyperthymic temperament $(p<0.001)$, anxious temperament $(p=0.002)$, BDI $(p$ $<0.001)$, BAI $(p<0.001)$, EMAQ-NE $(p<0.001)$ and EMAQ-NS $(p<0.001)$ scores differed between the three groups. Based on the Tukey test applied to the ANOVA analysis, only EMAQ-NS scores varied ( $p<$ 0.001) among the groups (Table 2).

According to hierarchical regression analysis (Table 3), it was found that the cyclothymic temperament scores accounted for $7.1 \%$ of the EMAQ-NE scores $(p<0.01)$ in obese participants (Step1). It was found that the cyclothymic, depressive, hyperthymic, irritable and anxious temperament scores accounted for $7 \%$ of the EMAQ-NE scores ( $p$ $<0.05$ ) in non-obese participants (Step 2). By adding BDI and BAI scores into this step it accounted for $9.6 \%$ of the EMAQ-NE scores (Step 3). It was also found that cyclothymic temperament scores accounted for $27.2 \%(p<0.001), 25.8 \%(p<0.001), 15.7 \%(\mathrm{p}<$ $0.001)$ of EMAQ-NS scores, in obese individuals with BED, obese and normal weight individuals respectively (Step 1). By adding depressive, hyperthymic, irritable and anxious temperament scores into this step, explanatory power increased to $29.1 \%(p<0.001), 32.6 \%(p<0.001), 36.8 \%(p<$ $0.001)$, respectively (Step 2). By adding BDI and BAI scores into this step, it was found that explanatory power increased to $75.9 \%(p<0.001), 77.6 \%(p<$ $0.001), 79 \%(\mathrm{p}<0.001)$, respectively (Step 3$)$. It was found that the cyclothymic temperament scores accounted for $5.9 \%$ of the EMAQ-PS scores $(p<0.01)$ in non-obese participants (Step1). By adding depressive, hyperthymic, irritable and anxious temperament scores into this step, it accounted for $10.9 \%$ of the EMAQ-PS scores (Step 2). By adding BDI and BAI scores into this step, it accounted for $11 \%$ of the EMAQ-PS scores (Step 3).

\section{DISCUSSION}

In our study, it was found that scores of appetite 
in negative emotions were higher in obese individuals with BED compared to obese individuals and it was higher in obese individuals compared to normal weight individuals. It was also determined that cyclothymic and hyperthymic features were more frequent in obese individuals with BED compared with obese and normal weigh individuals. Also, scores of appetite in negative situations in obese individuals with BED, obese and normal weight individuals were explained statistically significantly by bipolar features, depression, anxiety scores and especially cyclothymic features. In literature, it has been shown that emotional appetite couldbe an effective factor in the link between obesity and eating disorders (BED and bulimia nervosa) [3-5]. In this study, higher scores of appetite in negative emotions in obese individuals diagnosed with BED, compared with obese and normal weight individuals, is congruent with the results of the previous studies in literature. Levels of impulsivity, depressive symptoms and anger were found to high in individuals diagnosed with BED [29, 30]. It is therefore thought that appetite control in individuals diagnosed with BED is negatively affected by impulsive features and mental disorders. In this study findings of higher rates of cyclothymic and hyperthymic features in individuals diagnosed with BED compared with obese and normal weight individuals, support this aspect.

In obese individuals, comorbidities and high psychopathology rates may be confounding factors in the relationship between emotional appetite and BMI. In other words, the low confounding impact of comorbidities and psychopathological features in normal weight individuals may increase the appearance of the relationship between BMI and emotional appetite. In this study, a significant positive correlation was found between depressive temperament features and BMI values of obese individuals with BED. It is argued that depressive temperament could facilitate the development of bipolar disorders and depressive disorders [31] and high negative emotionality and low persistence in childhood could cause alterations in appetite and nutrition in individuals [32]. For this reason, there may be a relationship between BMI of individuals with BED and depressive temperament features. So, in order to understand the relationship between obesity and depressive disorders, more research on stable depressive features would be conducted.

The correlation between temperament features other than hyperthymic features and scores of appetite in negative situations in obese individuals with BED, obese and normal weight individuals implies that appetite in negative situations can be affected by negative mood in obese and normal weight individuals. Relationship between scores of appetite in positive emotions, negative emotions, positive situations and bipolar features, anxiety and depression differed among the three groups. Scores of appetite in negative emotions increased as bipolar features (except hyperthymic features) increasedin only obese patients. This may suggest that appetite in negative emotions in obese individuals with BED is associated with more diverse variables.

In obese individuals with lively, energetic and active characteristics, the need to eat in relation to positive emotions and situations may be lesser. The need for reward-seeking behaviors in relation to positive situations and emotions may also be less in obese individuals and they may be controlling their appetites better. Since higher frequencies of impulsive features in obese individuals compared with normal weight individuals were reported and obese individuals with ADHD were evaluated to be affected more in psychopathological features [33]. Our findings show that as cyclothymic, irritable and anxious features increased, appetite in relation to positive situations is decreased in normal weight individuals, however remain unchanged in obese with BED and obese individuals. This indicates that appetite in relation to positive situations can be affected by cyclothymic, irritable and anxious features in normal weight individuals whereas not in obese with BED and obese individuals.

In this study, only $2 \%$ of participants applying for bariatric surgery were diagnosed with bipolar disorder. In other previous studies, in individuals applying for bariatric surgery, bipolar diagnosis was found at different rates of 6-91.7\% [17-19]. It is stated that the incidence of bipolar disorder may change with age [34]. Therefore, despite the results of cross-sectional studies, the life time prevalence of bipolar disorder may increase in obese individuals. Therefore, in order to evaluate bipolar disorder diagnosis accurately in obese individuals, it may be essential to evaluate factors that affect the frequency such as age, sex and 
culture. In addition to this, in studies reporting higher rates of bipolar disorder, establishing the diagnosis of bipolar disease and features by scales instead of interviews by clinicians suggests the methodological limitations in these studies. In our study, bipolar disorder diagnosis in obese individuals applying for bariatric surgery was evaluated by clinical interview. Our study findings of bipolar frequency was similar to that of normal population and bipolar features (cyclothymia and hyperthymia scores) that may increase the risk of bipolar disorder were more frequent in obese individuals.

Nolan et al. [2] suggested that appetite can be affected by certain negative (when under pressure, after a heated argumen etc.) and positive (when falling love, after receiving good news) situations. Besides that, the emotions of individuals with cyclothymic features were determined to change rapidly during the day without any obvious reason (I get sudden shifts in mood and energy, my mood often changes for no reason) [22]. The increasing power of cyclothymia in explaining scores of appetite in negative situations towards normal weight (15.7\%), obese $(25.8 \%)$ and obese $(27.2 \%)$ individuals indicates that the cyclothymic features of obese individuals with BED make them more susceptible to negative situations. Another data supporting this finding is that after adding hyperthymic, anxious, irritable and depressive temperament scores to the model, the power to explain the negative situations score changed in normal weight $(36.8 \%)$, obese $(32.6 \%)$ and obese individuals with BED (29.1\%). In other words, it can be suggested that cyclothymic features are primary in explaining appetite in negative situationsin obese individuals with BED, whereas other bipolar features are effective in obese and normal weight individuals. Furthermore, in the last model (Table 3), bipolar features, depression and anxiety can be considered to be effective at high and similar levels $(79 \%, 77.6 \%, 75.9 \%$, respectively) in explaining appetite in negative situations in normal weight, obese and obese individuals with BED, respectively.

\section{Limitations}

Limitation of this study is that the number of participants is relatively small and the number of male participants are lower than female. Another limitation is the assessment of depression, anxiety and bipolar features by self-administered scales. This could affect data of the study. Another limitation is that the data of the study are only generalizable to obese individuals $(\mathrm{BMI}>34.9)$ applying for bariatric surgery.

\section{CONCLUSION}

As a result of this study, it can be concluded that scores of appetite in negative situations may be affected by cyclothymic features in obese individuals with BED, in obese and normal weight individuals. Depression and anxiety symptoms are effective factors in explaining scores of appetite in negative situations of obese individuals with BED, obese and normal weight individuals.

\section{Conflict of interest}

The authors disclosed no conflict of interest during the preparation or publication of this manuscript.

\section{Financing}

The author disclosed that they did not receive any grant during conduction or writing of this study.

\section{Acknowledgements}

The authors thank everyone who provided assistance for the study including medical secretaries.

\section{REFERENCES}

[1] Geliebter A, Aversa A. Emotional eating in overweight, normal weight, and underweight individuals. Eat Behav 2003;3:341-7.

[2] Nolan LJ, Halperin LB, Geliebter A. Emotional Appetite Questionnaire. Construct validity and relationship with BMI. Appetite 2010;54:314-9.

[3] Masheb RM, Grilo CM. Emotional overeating and its associations with eating disorder psychopathology among overweight patients with binge eating disorder. Int J Eat Disord 2006;39:141-6.

[4] Eldredge KL, Agras WS. Weight and shape overconcern and emotional eating in binge eating disorder. Int $\mathrm{J}$ Eat Disord 1996:19;73-82.

[5] Ricca V, Castellini G, Fioravanti G, Sauro CL, Rotella F, Ravaldi $\mathrm{C}$, et al. Emotional eating in anorexia nervosa and bulimia nervosa. Compr Psychiatry 2012;53:245-51.

[6] Turan Ş, Poyraz CA, Özdemir A. Binge Eating Disorder. Curr Approach Psychiatry 2015;7:419-35.

[7] Pinaquy S, Chabrol H, Simon C, Louvet JP, Barbe P. 
Emotional eating, alexithymia, and binge eating disorder in obese women. Obesity 2003;11:195-201.

[8] Gianini LM, White MA, Masheb RM. Eating pathology, emotion regulation, and emotional overeating in obese adults with binge eating disorder. Eat Behav 2013;14:309-13.

[9] Allison KC, Wadden TA, Sarwer DB, Fabricatore AN, Crerand CE, Gibbons LM, et al. Night eating syndrome and binge eating disorder among persons seeking bariatric surgery: prevalence and related features. Obesity 2006;14:77-82.

[10] Kalarchian MA, Marcus MD, Wilson GT, Labouvie EW, Brolin RE, LaMarca LB. Binge eating among gastric bypass patients at long-term follow-up. Obes Surg 2002;12:270-5.

[11] Chesler BE. Emotional eating: a virtually untreated risk factor for outcome following bariatric surgery. Sci World J 2012;2012:365961.

[12] Canetti L, Berry EM, Elizur Y. Psychosocial predictors of weight loss and psychological adjustment following bariatric surgery and a weight loss program: The mediating role of emotional eating. Int J Eat Disord 2009;42:109-17.

[13] Fischer S, Chen E, Katterman S, Roerhig M, BochierriRicciardi L, Munoz D, et al. Emotional eating in a morbidly obese bariatric surgery-seeking population. Obes Surg 2007; 17:778-4.

[14] American Psychiatric Association. Diagnostic and statistical manual of mental disorders (DSM-5®). American Psychiatric Pub 2013.

[15] McElroy SL, Kotwal R, KeckPE. Comorbidity of eating disorders with bipolar disorder and treatment implications. Bipolar Disord 2006;8:686-95.

[16] Martin K, Woo J, Timmins V, Collins J, Islam A, Newton D, et al. Binge eating and emotional eating behaviors among adolescents and young adults with bipolar disorder. J Affect Disord 2016;195:88-95.

[17] Duarte-Guerra LS, Coêlho BM, Santo MA, Lotufo-Neto F, Wang YP. Morbidity persistence and comorbidity of mood, anxiety, and eating disorders among preoperative bariatric patients. Psychiatry Res 2017;257:1-6.

[18] Alciati A, Gesuele F, Rizzi A, Sarzi-Puttini P, Foschi D. Childhood parental loss and bipolar spectrum in obese bariatric surgery candidates. Int J Psychiatry Med 2011;41:155-71.

[19] Grothe KB, Mundi MS, Himes SM, Sarr MG, Clark MM, Geske JR, et al. Bipolar disorder symptoms in patients seeking bariatric surgery. Obesity Surg 2014;24:1909-14.

[20] Arnow B, Kenardy J, Agras WS. The Emotional Eating Scale: The development of a measure to assess coping with negative affect by eating. Int J Eat Disord 1995;18:79-90.

[21] Michopoulos V, Powers A, Moore C, Villarreal S, Ressler KJ, Bradley B. The mediating role of emotion dysregulation and depression on the relationship between childhood trauma exposure and emotional eating. Appetite 2015;91:129-36. [22] Akiskal HS, Akiskal KK, Haykal RF, Manning JS, Connor PD. TEMPS-A: progress towards validation of a self-rated clinical version of the Temperament Evaluation of the Memphis, Pisa, Paris, and San Diego Autoquestionnaire. J Affect Disord 2005;85:3-16.

[23] Demirel B, Yavuz KF, Karadere ME, Şafak Y, Türkçapar MH. [The Emotional Appetite Questionnaire (EMAQ)'s Reliability And Validity and Relationship with Body Mass Index and Emotional Schemas]. J Cogn Behav Psychother Res 2014;3:171-81. [Article in Turkish]

[24] Beck AT, Ward CH, Mendelson M, Mock J, Erbaugh J. An inventory for measuring depression. Arch Gen Psychiatry 1961;4:561-71.

[25] Hisli N. [A study on the validity of Beck Depression Inventory]. Psikoloji Dergisi 1988;6:118-22. [Article in Turkish] [26] Beck AT, Epstein N, Brown G, Steer RA. An inventory for measuring clinical anxiety: psychometric properties. J Consult Clin Psychol 1988;56:893-7.

[27] Ulusoy M, Sahin NH, Erkmen H. Turkish version of the Beck Anxiety Inventory: psychometric properties. J Cogn Psychother 1998; 12:163-72.

[28] Vahip S, Kesebir S, Alkan M, Akiskal KK, Akiskal HS. Affective temperaments in clinically-well subjects in Turkey: initial psychometric data on the TEMPS-A. J Affect Disord 2005;85:113-25.

[29] Schag K, Schönleber J, Teufel M, Zipfel S, GielKE. Foodrelated impulsivity in obesity and Binge Eating Disorder -a systematic review. Obes Rev 2013;14:477-95.

[30] Fassino S, Leombruni P, Pierò A, Abbate-Daga G, Rovera GG. Mood, eating attitudes, and anger in obese women with and without binge eating disorder. J Psychosom Res 2003;54:559-66. [31] Akiskal HS, Akiskal K. Cyclothymic, hyperthymic, and depressive temperaments as subaffective variants of mood disorders. American Psychiatric Press Review of Psychiatry 1992;11:43-62.

[32] Martin GC, Wertheim EH, Prior M, Smart D, Sanson A, Oberklaid F. A longitudinal study of the role of childhood temperament in the later development of eating concerns. Int $\mathrm{J}$ Eat Disord 2000;27:150-162.

[33] Taymur I, Budak E, Onen S, Bicer B, Dilektaslı E, Cayci M, et al. The relationship between childhood and adult attentiondeficit-hyperactivity disorder and general psychopathological features in individuals who apply for bariatric surgery. Bariatr Surg Pract Patient Care 2016;11:116-22.

[34] Lin PI, McInnis MG, Potash JB, Willour V, MacKinnon DF, DePaulo JR, et al. Clinical correlates and familial aggregation of age at onset in bipolar disorder. Am J Psychiatry 2006;163:2406. 